\title{
Kinematics of the Circumnuclear Region of NGC 3351
}

\author{
A. I. Díaz ${ }^{1}$, G. F. Hägele ${ }^{1}$, M. V. Cardaci ${ }^{1,2}$, E. Terlevich ${ }^{3}$, \\ and R. Terlevich ${ }^{3}$ \\ ${ }^{1}$ Dpto. de Física Teórica, C-XI, Universidad Autónoma de Madrid, Cantoblanco, \\ 28049-Madrid, Spain \\ ${ }^{2}$ XMM-Newton Science Operation Centre, ESAC, ESA, PO Box 50727, 28080 Madrid, Spain \\ ${ }^{3}$ Instituto Nacional de Astrofísica, Óptica y Electrónica, Tonantzintla, Puebla, México
}

\begin{abstract}
Using high-resolution blue and far red spectra from the William Herschel Telescope, we have mapped the velocity field along three different slits in the central kiloparsec of the spiral galaxy NGC 3351. We have measured ionized gas (H $\beta$ and [OIII]) and stellar (near-IR Ca triplet) velocity dispersions for five individual Circumnuclear Star Forming Regions (CNSFRs) and the nucleus. Velocities and velocity dispersions in the red range were obtained using the crosscorrelation technique of Tonry \& Davis (1979AJ.84.1511) with a set of eleven templates, following the work by Nelson \& Whittle (1995.ApJSS.99.67) with the variations introduced by Palacios et al. (1997.A\&A.323.749). The gas velocities and velocity dispersions have been measured by fitting gaussians to the emission lines $(\mathrm{H} \beta$ and [OIII] $\lambda 5007 \AA)$ using three different suitable continua chosen by visual inspection and taking the average of the three results (Jiménez-Benito et al., 2000.MNRAS.317.907).

The rotation velocities derived for gas and stars are in very good agreement, although in some cases the gas seems to rotate slightly faster than stars. The turnover points of the rotational curves seem to be located at the same position than the star forming ring as found in other galaxies (Díaz, R. et al., 1999ApJ.512.623). The derived values are consistent with those previously obtained for the systemic velocity of NGC 3351 (Planesas et al., 1997.A\&A.325.81).

The velocity dispersions of the stars are always larger (by between 10 and $25 \mathrm{~km} / \mathrm{sec}$ ) than those derived from $\mathrm{H} \beta$ emission lines. In the cases in which it has been possible to use the Paschen emission lines, the results obtained are similar. The twice ionized oxygen, on the other hand, shows velocity dispersions comparable to the stellar ones or, in some cases, even larger. It is however important to point out that in these metal rich regions the [OIII] lines are very weak and they have a poor signal to noise ratio, then it is more difficult to accurately estimate the global kinematic behaviour from this emission line.

The difference between the behavior of ionized hydrogen and stars could be interpreted by assuming that the gas is confined to the disc and supported by rotation while the stars are mostly supported by dynamical pressure (see Pizzella et al., 2004.A\&A.424.447). However, the kinematics of ionized gas as mapped by [OIII], as we mentioned above, is similar to that of the stellar component.

We have applied the virial theorem to estimate the dynamical masses of the clusters and the galactic nucleus, assuming that the systems are gravitationally bound and spherically symmetric, and using sizes measured from the HST-WFPC2 image in the F606W filter (sizes between 2.6 and $5.1 \mathrm{pc}$ ). These values of the masses, estimated from stellar velocity dispersion measurements as mapped by the CaT lines, are in the range between $5.1 \times 10^{6}$ and $5.0 \times 10^{7} \mathrm{M}_{\odot}$ for the five studied CNSFRs and $3.6 \times 10^{7} \mathrm{M}_{\odot}$ for the nucleus of the galaxy, in the inner $23.4 \mathrm{pc}$.
\end{abstract}

Keywords. galaxies: star clusters, galaxies: individual (NGC 3351), galaxies: kinematics and dynamics 